\title{
Cognitive Ecology and Cognitive Agriculture - New Interdisciplinary Scientific Areas
}

\section{Vahram R Sargsyan*}

President of the International Academy of Neuroscience, L Orbeli Institute of Physiology, Yerevan, Republic of Armenia

*Corresponding Author: Vahram R Sargsyan, PhD, President of the International Academy of Neuroscience, L Orbeli Institute of Physiology, Yerevan, Republic of Armenia.

Received: May 23, 2019; Published: July 09, 2019

DOI: $10.31080 /$ ASNE.2019.02.0079

\section{Abstract}

The paper presents the practical significance and benefits of new interdisciplinary scientific fields - cognitive ecology and cognitive agriculture. Cognitive ethology and cognitive agriculture are based on the latest advances in neurogenetics and physiology, and are of strategic importance for the protection of the environment and the development of the agricultural sector. For the formation and development of cognitive ecology and agriculture played a significant role nano-model theory of the genome, modern understanding of the formation of higher nervous activity and the presence of biocommunication system in humans and other biological species. The presence of neuroplasticity in nature is also important.

Keywords: Cognitive Ecology; Cognitive Agriculture; Neurogenetics; Physiology; Neuroplasticity; Nano-Model Theory; Higher Nervous Activity; Biocommunication System

\section{Introduction}

Cognitive ecology and cognitive agriculture are new scientific areas that have arisen at the interface of neurobiology (neurogenetics and neurophysiology), physiology, ecology and agriculture. Over the past decades of human development, individually, various scientific fields have achieved great success. However, at this stage of development, great attention should be paid to the development of interdisciplinary research (theoretical and practical). Cognitive ecology is based on recent advances in neurogenetics (nano-model theory of genome functioning) [5], neurophysiology (modern understanding of the formation of higher nervous activity) [9] and human physiology (presence of the biocommunication system in humans) [10]. Cognitive ecology is of strategic importance for environmental protection. Cognitive agriculture is mainly based on the presence of the biocommunication system in humans, animals and plants, which creates a common biological field of our ecosystem. This biofield has a completely molecular structure and consists of free-living microorganisms and mobile genetic elements. Cognitive agriculture is aimed at ensuring the food security of mankind. Before we present the practical significance and benefit of these scientific areas, let us give a scientific definition of cognitive science, ecology, cognitive ecology and cognitive agriculture.
- Cognitive science: An interdisciplinary scientific direction, combining the theory of knowledge, cognitive psychology, neurophysiology, cognitive linguistics, nonverbal communication and the theory of artificial intelligence.

- Ecology: The science of the interactions of living organisms and their communities among themselves and with the environment. The modern interpretation of the concept of ecology is much broader than in the first decades of the development of this science. At present, environmental issues are most often mistakenly referred to as environmental issues.

- Cognitive ecology is an interdisciplinary scientific field that studies the relationship and interrelationship of the psyche ("inner world") of a person and the environment (living and inanimate nature). Cognitive ecology studies the mechanisms of formation of human higher nervous activity depending on the environment and changes in the human environment depending on the functioning of human higher nervous activity.

- Cognitive agriculture is an interdisciplinary scientific field that studies the interrelation and interrelationships of the psyche ("inner world") of a person on the one hand, and animals, plants, fungi, agricultural microorganisms on the other. Cognitive agriculture studies the mechanisms of formation of human behavior depending on the food supply of agricultural 
production and changes in yield and quality of agricultural products depending on the functioning of the human cognitive sphere.

- The agricultural insurance system throughout the world plays a major role in the development of cognitive agriculture.

- The value of cognitive ecology lies in the fact that it represents a scientifically based argument in favor of the possibility of controlling the habitat on the part of man. By changing his thinking (inner world/psyche) a person is able to actively change the outer world and therefore bears a huge responsibility before Nature.

- It is very important to take into account the latest scientific achievements in the field of neurogenetics, neurophysiology and human/animal/plant physiology, on the basis of which cognitive ecology and agriculture arose. Below we briefly present the results of our research in these areas.

\section{Nano-model theory of the functioning of the genome}

According to our nano-model theory of genome functioning, the DNA molecule stores biological information not only in the form of a genetic code consisting of a sequence of nucleotides, but also in the form of a spatial-structural organization. This means that the information component lies not only in the primary structure of the organization of DNA molecules, but also in the II and III structures. This is actually a kind of biological nano-layouts $[5,7,9]$.

A similar function in nature, and can carry out RNA molecules, as well as to some extent, and protein molecules.

DNA contains information about the structure of different types of RNA and proteins [1]. But this does not mean that the DNA molecule is not able to independently carry out numerous biological functions that ensure the functioning of living systems.

Almost all genes function on the principle of nano-layouts. However, based on the fact that many genes of the main genome are localized in the cell nucleus, and must function in the cytoplasm or outside the cell, so nature has created known modern biology transcription and translation processes. The protein has a volumetric structure (a certain form) due to its II, III, sometimes even IV structure. It is known, for example, that the protein enzyme has an active center, functioning on the principle of the key to the lock. It will have a certain functional activity depending on its shape. DNA molecule (its specific site - gene) also has II, and III structure, that is, it is not just a linear molecule consisting of nucleotides $[1,3]$.

Our nano-model theory of genome functioning perfectly reflects the numerous processes taking place both at the cellular and at the organism levels. Genes, functioning on the principle of nano- layouts are actually a kind of copy of the macrocosm. Depending on the adequacy of the reflection of the macrocosm at the cellular level, we can judge the level of quality of perception of information from the body. The well-known expression: "the Brain is in the World, and the World is in the Brain", becomes fully understandable thanks to the above scientific data.

Current understanding of the formation of higher nervous activity in humans

If we take into account that the organism (cell) has the main and acquired genome [5], this fact sheds light on many, currently unresolved scientific issues and, first of all, on aspects of the genetic level of the organism. In turn, it becomes clear how and by what molecular mechanisms the differentiation of cells in multicellular organisms in the process of individual development (ontogenesis) is carried out. Scientifically fully justified, for example, the emergence of highly specialized functions in the neurons of the human brain and the manifestation already at the organizational level of various functions of higher nervous activity, many of which even at the present stage of human development are considered "secrets" of science.

Therefore, it is not surprising that geneticists who study the human genome are struggling to find those genetic features that caused the increase in the brain and, perhaps, its more effective work. Special hopes are placed on the comparison of the human genome with the chimpanzee genome. This allows us to immediately exclude from consideration those $98 \%$ of the genome that are identical in our species. Somewhere out there, in the remaining two percent, the mystery of human uniqueness is encrypted. It remains to understand where and how.

Behavior and mental abilities of the person are at qualitatively new level in comparison with monkeys. It is reasonable to assume that these differences are genetic in nature.

As a result of serious studies, scientists have proved that the origin of man was not observed universal and large-scale accumulation of amino acid changes in genes involved in the nervous tissue $[2,12]$.

According to the proposed classification of the genome on the main and acquired (based on our viral theories [6]) and nano-model theory of genome functioning [5] all this can be explained very logically and scientifically justified. The fact is that modern classical genetics study only the main genome of the body, that is, genes derived from the parent germ cells (egg and sperm). However, for the functioning of highly specialized cells (such as, for example, brain neurons) - those genes that were obtained from parents by 
vertical transmission (from germ cells as a result of the formation of a zygote) will not be enough. According to our viral theories, for the full perception of information, the formation of memory and the functioning of the somatic and autonomous nervous system, the body in the process of ontogenesis must additionally obtain a certain set of genes by horizontal gene transfer. This normally occurs in the perinatal and postnatal periods of individual development of the body. In order for most of the highly specialized cells in the human body (or other multicellular organism) began to fully perform their intended functions - it is not enough to "turn on" (expression) certain groups of genes and "turn off" other groups of genes of the main genome. If it were that simple, geneticists would have found a lot of genes from the main human genome, which are inherent only to us (humans) and distinguish us, for example, from monkeys. The fact that man in terms of its development is quite superior to other species of animals, in my opinion there is no doubt. And these differences are due to the receipt of additional genes already in the process of human ontogenesis. The basic human genome only creates the prerequisites (favorable conditions) for the implementation of this important process and only a small number of genes are necessary for this.

\section{Brain plasticity and the plasticity of the genome}

According to the above genome of the body - it is actively and dynamically developing system throughout the period of ontogenesis. To make this thesis more convincing, see the analogy with the plasticity of the human brain in our scientific article published in 2018 [9].

Scientists with a little delay, but still came to the conclusion about the existence of neuroplasticity [4] in nature. Why not accept the fact about the plasticity of the genome (the processes of occurrence/destruction of genes in the process of ontogenesis of the body) and with the help of this understanding of the fundamental biological processes to explain the many processes occurring in nature and are "mysteries" of science. I suggest that geneticists do not make "mistakes" neuroscientists and timely review and determine the question of the plasticity of the genome of the body, which will certainly have a huge impact on the development of biological Sciences and numerous practical areas of knowledge.

\section{Biocommunication system}

On the basis of the latest achievements in genetics and cell biology, as well as the correct understanding of the true role and functions of viruses in nature, it became possible to revise the basics of human physiology and anatomy. This will make a scientific brea- kthrough in the field of biology and medicine. There is an urgent need to understand and accept the presence of a new human body system - biocommunication system (BS), which, along with the nervous and endocrine systems is the most important regulatory and integrating system of the human body and other biological species. Human BS is responsible for the formation of higher nervous activity and therefore the study of this system of the body will help to understand the mechanisms of formation of various diseases, as well as environmental problems.

For a full understanding of the subsequent part of the scientific article it is necessary to get acquainted with viral [6] and genetic [5] theories, as well as with the new classification of the genome. And only after this begin to study BS rights.

Biocommunication system [10] is present in all multicellular organisms, but different species are developed to some extent. To date, all known species are the most complex BS a person has. The cause of many diseases is the destruction or improper functioning of the BS $[8,11]$. Neurodegenerative and oncological diseases are a vivid example of the destruction of human BS.

\section{Structure of human BS}

The main components of the human BS are:

- Biocommunication (DNA and RNA). Biocommunication - migrating organelles of eukaryotic cells.

- The microbiome - the totality of genes, diversity of the microbiota (microflora) of different ecological niches. Microbiota includes several thousand types of mushrooms, eubacteria, archaea and viruses (biocommunication). This is the socalled bacterial human organ.

- Ventricles of the brain - cavities in the brain filled with cerebrospinal fluid.

- $\quad$ Spinal canal - longitudinal canal, located in the gray matter of the spinal cord, at the top it connects with the cavity of the fourth ventricle of the brain, and at the bottom forms a kind of expansion in the form of a final ventricle.

- Limfatic system - anatomic liquor way of elimination of waste products of the tissues of the Central nervous system of mammals.

- Blood and lymph vessels, nerves and transport infrastructure for biocommunication.

- Terminals biocommunication (viral terminal) - edge part of the Central Department of BS which provides the relationship of the visible body of the organism with the external environment. 
- Free-living microorganisms (viruses/biocommunication) microbiome, divorced from the physical-visible body. They make up the human aura and are the physical-invisible body of the human body. Man is actually an ecosystem inhabited not only by cells derived from the zygote, but also by numerous representatives of the microbiome.

\section{Departments of human BS}

- Central BS - biocommunicators and the microbiome within the physical visible body of the body; the ventricles of the brain; the spinal canal; glimfatik system; blood and lymphatic vessels, nerves; terminals of biocommunicators.

- Peripheral BS - free-living microorganisms (viruses/biocommunicators) of microbiome, divorced from the physical-visible body. They are the physical-invisible body of the human body, as they carry genetic information about the body.

\section{Human BS functions}

Human BS performs many vital functions. It is closely related to the nervous, endocrine, immune and other systems of the human body. Anatomically, the BS can be found within all other body systems. In fact, anywhere where there is an active biocommunication can function biocommunication system.

1. Function of the Electromagnetic Reception: This ensures remote communication with the environment. The main natural sources of electromagnetic radiation in nature are the Sun, the Earth and other celestial bodies. The human BS ensures the connection of the human body with the specified celestial bodies, ensures the full integration of man with the environment.

2. Function of Biocommunication: One of the most important functions of a human BS. Thanks biocommunication there is effective communication between the various biological species in nature. This is a biological way of communication, when information from one organism to another is transferred in the form of ready-made bio-nano-models, with molecules of DNA, RNA and proteins. The function of biocommunication has two varieties - biological attack and bio-humanitarian support.

3. Function of the Signal transduction: Biocommunicators within the physical body of a person provide well-established work of trillions of cells of the body. It is known that the signaling is provided not only physical and chemical interactions between cells of a multicellular organism, and the biological by - biocommunicators. Violation of this function leads to the formation of cancer and neurodegenerative diseases (e.g. Alzheimer's disease).

4. The function of monitoring the energy system of cells: It biocommunicators responsible for many energy processes in the cells of the human body. Violation of this function leads to dysfunction of the mitochondria and oxidative stress. This is one of the causes of Alzheimer's disease and many other diseases.

5. The function of monitoring of the immune system: Biocommunication actively managing numerous cells of the human immune system and cells of the human microbiome. This ensures the control and safe functioning of the human body, and its protection from aggressors from the environment.

6. Function of higher nervous activity: This is a very important function and is based on the perception of information, memory formation and functioning of the human somatic nervous system. In other words, the sensorimotor activity of the person. All this is achieved with the active participation of biocommunicators.

7. Function of control of activity of various systems of an organism: Biocommunicators control the functioning of the autonomic nervous system, endocrine, cardiovascular, reproductive, digestive, respiratory, excretory and other systems. This ensures the integration of all parts of the body into a single whole.

8. The function of human development. Biocommunication responsible for the processes of plasticity of the genome of the organism and the neuroplasticity (brain plasticity) [8,9]. This determines the development of man and it creates the basis for the process of evolution of man and the organic world.

\section{Human BS segments}

BS has the following segments:

1. Segment of the present: This is the area of human consciousness where his real actions take place. They are based on information from numerous sensory receptors and the human body

2. Segment of the past: This segment is responsible for longterm human memory. It characterizes a person's personality.

3. Segment of the future: A place where a person makes plans for the future.

Thus, it is necessary to continue to study human BS and on the basis of scientific data to develop a new scientific direction - cognitive ecology and cognitive agriculture 
In fact, cognitive ecology provides an opportunity to improve the living environment for billions of people around the world. Cognitive agriculture provides an opportunity to increase the yield and quality of crops and livestock efficiency, as well as contributes to the development of veterinary medicine. It is only necessary to conduct appropriate propaganda among young people and other groups of the population about the important role of the formation of their "inner world" and effective biocommunication. The purposeful formation of the "inner world" of man is the key to improving the environmental and agricultural situation on our Planet. It is necessary to scientifically and logically justified to present to people the importance of hygiene of their thoughts, which will certainly be the key to their perfect health and improve the environment (Agroecology) in the World.

\section{Bibliography}

1. Alberts B., et al. "Molecular biology of the cell". (4th edn). New York: Garvard Science, USA (2002).

2. Khaitovich P., et al. "Parallel patterns of evolution in the genomes and transcriptomes of humans and chimpanzees". Science 309 (2005): 1850-1854

3. Klug W., et al. "Essentials of Genetics". (9th edn) (2015): 608.

4. Norman D. "Brain plasticity: Stunning facts about how thoughts are capable of changing the structure and function of our brain". (2010): 544

5. Sargsyan VR. "The main and acquired genome. Nano-model theory of genome functioning". "International Science Project". (2018): 8-13.

6. Sargsyan VR. "The true place and role of viruses in nature. Viruses - migrating cell organelles". International Science Project (2018): 4-8.

7. Sargsyan VR. "New Scientific Theories - The Base for Creating Perspective Methods of Treating Different Diseases". Journal of Brain Neuroscience 3 (2019): 008.

8. Sargsyan VR. "Formation of Higher Nervous Activity in Human and Autism Spectrum Disorder". Acta Scientific Women's Health 1.1 (2019): 22-24.

9. Sargsyan VR. "Formation of Human Nervous Activity and New Biological Theories". Journal of Brain Neuroscience 2 (2018): 004.

10. Sargsyan VR. "Human Biocommunication System and New Health Care System". Alzheimer's Research and Therapy 2.1 (2019): 000105.
11. Sargsyan VR. "Mechanisms of formation of oncological and neurodegenerative diseases on the basis of viral theory of signal transduction". Med Crave. Advances in Obesity Weight Management and Control 9.1 (2019).

12. Squire L., et al. "Fundamental Neuroscience". (3rd edn), USA (2008).

Volume 2 Issue 8 August 2019

(C) All rights are reserved by Vahram R Sargsyan. 